First Draft

\title{
Stochastic Cosmology and the Vacuum Energy Parameter
}

\author{
Ervin Goldfain
}

Research Scholar, Ronin Institute, Montclair, New Jersey 07043

Email: ervin.goldfain@ronininstitute.org

\begin{abstract}
The Vacuum Energy Parameter (VEP) of standard cosmology $\left(\Omega_{\Lambda}\right)$ denotes the fraction of the critical density attributed to the accelerated expansion of the Universe. Astrophysical evidence sets the numerical range of VEP at $\Omega_{\Lambda}^{\text {exp }}=0.692 \pm 0.012$, yet the root cause of these values is currently unknown. Drawing from the stochastic interpretation of early-Universe cosmology, we develop here a derivation of the VEP based on classical diffusion theory and the Langevin equation. Predictions are shown to be in reasonable agreement with observations.
\end{abstract}

Key words: FRW model, accelerated expansion, Vacuum Energy parameter, stochastic cosmology, Langevin equation.

\section{Introduction}

It is known that the Friedmann-Robertson-Walker (FRW) model is based on the cosmological principle, according to which matter distribution in the large-scale structure of the Universe is homogeneous and isotropic [1]. Besides homogeneity and isotropy, the FRW model implicitly assumes that cosmological processes are governed by 
deterministic laws excluding - by default - concepts such as randomness and probability distributions.

Over the years, several studies have advocated extending the basis of FRW cosmology, arguing that a stochastic description may be better equipped to model the early evolution of the Universe [3-6]. We fully subscribe to this viewpoint and go even further in suggesting that stochastic cosmology is, in fact, an integral part of complex dynamics. The goal of complex dynamics is to explore the evolution of large interacting ensembles that are out-of-equilibrium and capable of sustaining a wide spectrum of collective behaviors. As the dynamics of complex systems includes non-deterministic processes, it provides a natural foundation for the ideas of stochastic cosmology. Our approach starts from the premise that the cosmic fluid density of the FRW model may be mapped to a classical scalar field. Following the philosophy of stochastic cosmology, we show that behavior of this field matches the dynamics of a damped harmonic oscillator in contact with a reservoir of random fluctuations. Universe expansion supplies the damping mechanism and is embodied in the Hubble parameter. Setting the gravitational self-interaction scale as the upper bound energy of the scalar field leads to predictions in reasonable agreement with observations.

The paper is partitioned in the following way: next section delves into the stochastic interpretation of early Universe cosmology while section 3 defines the set of assumptions and conventions underlying our work. The construction of the Langevin equation and the derivation of the VEP form the object of sections 4 and 5. Concluding remarks and follow up developments are discussed in the last section. 


\section{Stochastic interpretation of early Universe cosmology}

The mass dimension of fluid density in natural units is given by

$$
[\rho]=\frac{m}{V}=\frac{m}{L^{3}}=L^{-4}=\mu^{4}
$$

in which $\mu=[L]^{-1}$ is an arbitrary mass scale. The FRW fluid density may be associated with a classical scalar field as in

$$
\varphi \Leftrightarrow \rho^{1 / 4}
$$

whose mass dimension in $3^{+1}$ spacetime is (see [2] for example)

$$
[\varphi]=L^{-1}=\mu
$$

It is important to draw an upfront distinction between (2) and the hypothetical fields of cosmic inflation, such as "inflaton", "dilaton" or "quintessence". These fields supplement the standard framework of cosmology but do not share common roots with the fluid density concept of the FRW model. Despite their difference in physical nature, both (1) and the postulated fields of inflationary cosmology satisfy the scalar field equation [1]

$$
\ddot{\varphi}+\lambda_{H} \dot{\varphi}+\frac{\partial V(\varphi)}{\partial \varphi}=0, \quad \lambda_{H}=3 H
$$

where $V(\varphi)$ stands for a generic potential function. To understand why this is the case, consider the Lagrangian density of any scalar field in curved spacetime [1]

$$
L=\frac{1}{2} g^{\mu \nu} \frac{\partial \varphi}{\partial x^{\mu}} \frac{\partial \varphi}{\partial x^{\nu}}-V(\varphi)
$$


The corresponding field equation is

$$
\frac{1}{\sqrt{-g}} \frac{\partial}{\partial x^{\mu}}\left(-\sqrt{-g} g^{\mu \nu} \frac{\partial \varphi}{\partial x^{\nu}}\right)+\frac{\partial V(\varphi)}{\partial \varphi}=0
$$

Space derivatives can be safely ignored in comparison with the time derivative, under the plausible assumption that the field is spatially homogeneous. If, in addition, the geometry is close to being spatially flat, the line element may pe cast in a form compatible with the FRW cosmology, i.e.

$$
d s^{2}=d t^{2}-a(t)^{2}\left(d r^{2}+r^{2} d \Phi\right)
$$

It follows from these considerations that $\sqrt{-g}=a^{3}$. Then, by (6) and (7), one recovers the field equation (4).

It is not unreasonable to think that (4) may be generalized to a scenario including random fluctuations present in the evolution of the early Universe. The most straightforward path to this scenario consists of adding a "thermal-like noise" term in the right-hand side of (4). This leads to

$$
\ddot{\varphi}+\lambda_{H} \dot{\varphi}+\frac{\partial V(\varphi)}{\partial \varphi}=\eta(t)
$$

For simplicity, we take the statistics of the noise term to be delta correlated as in

$$
\left\langle\eta(t) \eta\left(t^{\prime}\right)\right\rangle=D \delta\left(t-t^{\prime}\right)
$$

where $D$ denotes the strength of noise correlations. In line with classical diffusion theory in a harmonic potential, we posit that $V(\varphi)$ is a quadratic function 


$$
V(\varphi)=\frac{1}{2} \Omega^{2} \varphi^{2}
$$

so that

$$
\frac{\partial V(\varphi)}{\partial \varphi}=\Omega^{2} \varphi
$$

Next section defines the main set of assumptions and conventions used below in the analysis of (8).

\section{Assumptions and conventions}

A1) In section 5, both scalar field and time parameters are cast in dimensionless form and written as $x^{0}$, where $x=(\varphi, t)$. Carrying out this operation means normalizing $x$ with the appropriate powers of the mass scale $\mu$. The rationale for the most natural choice of $\mu$ in the context of our paper is also given in section 5 .

A2) To fix the initial conditions, we posit that the amplitude of the scalar field starts from an arbitrarily chosen lower value and develops larger values at later times. Hence, we assume

$$
\varphi\left(t_{0}\right)<<\varphi(t) \text { for } t_{0}<t
$$

A3) The vacuum of the FRW model evolves from a regime of out-of-equilibrium fluctuations in the early Universe and settles into a stationary state $\rho_{\Lambda}=-p_{\Lambda}$ in the longtime limit $t_{\infty}>>t>t_{0}$. This is a state of full thermal equilibrium where expectation and instantaneous parameter values coincide viz. 


$$
\left\langle\rho_{\Lambda}\right\rangle=\rho_{\Lambda}=\rho\left(t_{0}\right)
$$

\section{Vacuum state and the stationary regime of the Langevin equation}

The evolution of field expectation value in the long-time limit of classical diffusion theory $\left(t>>t_{0}\right)$ satisfies (cf. the Appendix section)

$$
\frac{d\langle\varphi\rangle}{d t}=-\frac{\langle\varphi\rangle}{\tau}
$$

and leads to the exponential decay equation

$$
\langle\varphi(t)\rangle=\left\langle\varphi\left(t_{0}\right)\right\rangle \exp \left(-\frac{t}{\tau}\right)
$$

Here, $\tau=\lambda_{H}^{-1}$ plays the role of a time constant associated with the Langevin equation (8). By analogy with (13) and (14), one may define a time constant which is formally equivalent to $\tau$ and refers to the instantaneous realization of the field variable. Explicitly, this equivalent time constant is defined by

$$
\tau_{\text {equiv }}=\frac{\int_{t_{0}}^{t} \frac{d \varphi(s)}{d s} d s}{\dot{\varphi}(t)}
$$

so that

$$
\varphi(t)=-\tau_{\text {echiv }} \dot{\varphi}(t)+\varphi\left(t_{0}\right)
$$

Direct substitution of (16) in (8) - (11) and on account of A2) gives 


$$
\ddot{\varphi}+\left(\lambda_{H}-\lambda_{c, H}\right) \dot{\varphi}=\eta(t)
$$

in which

$$
\Omega^{2} \varphi=-\Omega^{2}\left(\tau_{\text {equiv }} \dot{\varphi}\right) \Rightarrow \lambda_{c, H}=-\Omega^{2} \tau_{\text {equiv }}
$$

It is instructive to evaluate next the analogy between (17) and the local energy conservation equation of the FRW cosmology. To this end, we start from the stochastic model of early Universe discussed in [9], whereby the state parameter $\omega$ is assumed to be a fluctuating entity. The conservation equation reads

$$
\dot{\rho}+\lambda_{H}[1+\omega(t)] \rho=0
$$

in which $p(t)=\omega(t) \rho(t)$ represents the time-dependent generalization of the equation of state. Differentiating (19) leads to

$$
\ddot{\rho}+\lambda_{H}[1+\omega(t)] \dot{\rho}+\lambda_{H} \dot{\omega}(t) \rho=0
$$

Comparative inspection of (17) and (20) reveals the following mapping

$$
\lambda_{H}-\lambda_{c, H} \Leftrightarrow \lambda_{H}[1+\omega(t)]
$$

$$
\lambda_{H} \dot{\omega}(t) \Leftrightarrow \eta(t)
$$

The limit $\lambda_{H}-\lambda_{c, H} \rightarrow 0$ corresponds to fixing the state parameter at $\omega=-1$, so that $\dot{\omega}=0$ and $\eta(t)=0$. By A3), these settings recover the equilibrium conditions of the vacuum regime in standard FRW cosmology [1]. 
In closing this section, we mention that there are two key relationships linking the coefficient $D$ of (9), the damping coefficient $\lambda_{H}-\lambda_{c, H}$ of (17) and the eigenfrequency $\Omega$ of the harmonic potential (10) [7]:

a) The first relationship follows from the fluctuation-dissipation theorem of thermal equilibrium at long times $\left(t>>t_{0}\right)$, and it requires

$$
D \propto\left(\lambda_{H}-\lambda_{c, H}\right) k_{B} T
$$

where $k_{B}$ is the Boltzmann constant and $T$ the temperature.

b) The second relationship stems from the equipartition theorem of Thermodynamics and takes the form

$$
\left\langle\dot{\varphi}^{2}(t)\right\rangle_{e q}=k_{B} T
$$

\section{Derivation of the Vacuum Energy Parameter (VEP)}

To further proceed with our derivation, we recall that the Einstein-Hilbert action in the absence of matter or radiation takes the form $[1,10]$

$$
S=\frac{1}{16 \pi G_{N}} \int d^{4} x \sqrt{-g} R=M_{G}^{2} \int d^{4} x \sqrt{-g} R
$$

where $R$ represents the scalar curvature, $G_{N}$ is Newton's constant and

$$
M_{G}=\left(16 \pi G_{N}\right)^{-1 / 2}
$$

sets the natural scale of gravitational self-interaction, induced by gravitation in empty space. Hence, by assumption A1), the relevant choice for the mass scale is 


$$
\mu=M_{G}
$$

The dimensionless parametrization of the field and its time rate are then given by

$$
\begin{gathered}
\varphi^{0}=\frac{\varphi}{M_{G}} \\
t^{0}=\left(\lambda_{H}-\lambda_{c, H}\right) t
\end{gathered}
$$

Using (28) and (29) leads to

$$
\dot{\varphi}^{0}=\frac{d \varphi^{0}}{d t^{0}}=\frac{1}{\left(\lambda_{H}-\lambda_{c, H}\right) M_{G}} \frac{d \varphi}{d t}=\frac{1}{\left(\lambda_{H}-\lambda_{c, H}\right) M_{G}} \dot{\varphi}
$$

By (28)-(30) and on account of (10) and (18), the expectation values for the kinetic and potential terms for the dynamics of the scalar field can be presented as

$$
\begin{aligned}
& \left\langle T_{k i n}\right\rangle=\frac{1}{2}\left\langle\dot{\varphi}^{2}\right\rangle=\frac{1}{2}\left(\lambda_{H}-\lambda_{c, H}\right)^{2} M_{G}^{2}\left\langle\left(\dot{\varphi}^{0}\right)^{2}\right\rangle \\
& |\langle V(\varphi)\rangle|=\frac{1}{2} \Omega^{2}\left\langle\varphi^{2}\right\rangle=\frac{1}{2} \frac{\lambda_{c, H}}{\tau_{\text {equiv }}} M_{G}^{2}\left\langle\left(\varphi^{0}\right)^{2}\right\rangle
\end{aligned}
$$

The expectation values for the energy density and pressure associated with the scalar field assume the form [1]

$$
\begin{aligned}
& \langle\rho\rangle=\left\langle T_{k i n}\right\rangle+\langle V(\varphi)\rangle \\
& \langle p\rangle=\left\langle T_{k i n}\right\rangle-\langle V(\varphi)\rangle
\end{aligned}
$$


Demanding compliance with the equation of state for the vacuum in the FRW model yields the following constraint expressed in terms of expectation values

$$
\langle p\rangle=-\langle\rho\rangle \Rightarrow\left\langle T_{k i n}\right\rangle=\left\langle\dot{\varphi}^{2}\right\rangle=0
$$

By (23) and (31a), (33) implies two equilibrium conditions for asymptotically long times $t=t_{\infty} \rightarrow \infty$

$$
\lambda_{H}-\lambda_{c, H} \rightarrow 0, D \rightarrow 0
$$

and

$$
|\langle V(\varphi)\rangle|=\frac{1}{2} \frac{\lambda_{H}}{\tau_{\text {equiv }}} M_{G}^{2}\left\langle\left(\varphi^{0}\right)^{2}\right\rangle
$$

It is instructive to confirm, at this point, that (33) is consistent with (24) near thermal equilibrium, when the Universe temperature drops close to zero in the long-time limit [7]

$$
\left\langle\dot{\varphi}^{2}(t)\right\rangle_{e q}=k_{B} T \Rightarrow\left\langle\dot{\varphi}^{2}\left(t_{\infty}\right)\right\rangle \rightarrow 0
$$

Referring now to the Appendix equation (A7), a plausible hypothesis is that the time constant and instantaneous time constants defined in (13) to (15) become equal near or at thermal equilibrium. It follows that,

$$
\tau_{\text {equiv }}=\lambda_{c, H}^{-1}=\lambda_{H}^{-1} \text { for } t \rightarrow t_{\infty}
$$

It is also reasonable to expect that, in the asymptotic vacuum state defined by (33), the energy carried by the scalar field $\varphi$ is upper limited by the gravitational scale $M_{G}$. On 
account of these considerations and by (28), (35) and (37), one obtains the following stationary expectation value of the vacuum density

$$
\left\langle\rho^{0}\right\rangle=1 \rightarrow\left\langle\rho_{\Lambda}\right\rangle=\langle V(\varphi)\rangle=\frac{9}{2} H^{2} M_{G}^{2}
$$

The critical density of the FRW model is given by (in natural units $c=1$ ),

$$
\rho_{c}=\frac{3 H^{2}}{8 \pi G_{N}}=6 H^{2} M_{G}^{2}
$$

Dividing (38) to (39) produces the main result of our paper, namely,

$$
\Omega_{\Lambda}=\frac{\left\langle\rho_{\Lambda}\right\rangle}{\rho_{c}}=.75
$$

in reasonable agreement with current astrophysical data $\left(\Omega_{\Lambda}^{\mathrm{exp}}=0.692 \pm 0.012\right)$. Recall that, by assumption A3), the instantaneous and expectation values of the vacuum density $\rho_{\Lambda}$ are set to be identical.

\section{Conclusions and outlook}

Our work has developed from the premise that the cosmic fluid density of the FRW model may be mapped to a classical scalar field. Following in the footsteps of stochastic cosmology, we have found that the behavior of this field corresponds to the dynamics of a damped harmonic oscillator in contact with a reservoir of random fluctuations. In this picture, Universe expansion supplies the damping mechanism as embodied in the Hubble 
parameter. Setting the gravitational self-interaction scale as the upper bound energy of the scalar field yields a VEP prediction in reasonable agreement with observations.

In line with [11-12], a sequel to this work will attempt bridging the gap between stochastic cosmology and self-organized criticality (SOC). The plan is to show that SOC sheds new light on the inner workings of the early-Universe cosmology [13].

\section{$\underline{\text { APPENDIX }}$}

The goal of the Appendix section is to recover (13) from the classical diffusion theory applied to Brownian motion [8]. The evolution of the field expectation value is described by

$$
\langle\varphi(t)\rangle=\varphi\left(t_{0}\right)+\frac{\dot{\varphi}\left(t_{0}\right)}{\lambda_{H}}\left[1-\exp \left(-\lambda_{H} t\right)\right]
$$

Assumption (12) reduces (A1) to the following approximation

$$
\langle\varphi(t)\rangle=K\left[1-\exp \left(-\lambda_{H} t\right)\right]
$$

where

$$
K=\frac{\dot{\varphi}\left(t_{0}\right)}{\lambda_{H}}
$$

Differentiating (A2) yields

$$
\frac{d}{d t}\langle\varphi(t)\rangle=\dot{\varphi}\left(t_{0}\right) \exp \left(-\lambda_{H} t\right)
$$

By (A2), (A4) becomes 


$$
\frac{d}{d t}\langle\varphi(t)\rangle=\dot{\varphi}\left(t_{0}\right)-\lambda_{H}\langle\varphi(t)\rangle
$$

A suitable linear shift of the form

$$
\langle\varphi(t)\rangle_{s}=\langle\varphi(t)\rangle-K=\langle\varphi(t)\rangle-\frac{\dot{\varphi}\left(t_{0}\right)}{\lambda_{H}}
$$

turns (A4) into

$$
\frac{d}{d t}\langle\varphi(t)\rangle_{s}=-\frac{1}{\tau}\langle\varphi(t)\rangle_{s}
$$

which recovers (13) upon the identification

$$
\tau=\lambda_{H}^{-1}=\frac{1}{3 H}
$$

\section{$\underline{\text { References }}$}

1. Peebles P. J. E, "Principles of Physical Cosmology”, Princeton Univ. Press, 1993.

2. Ryder L. H., “Quantum Field Theory”, Cambridge Univ. Press, 1989.

3. Available at the following site

https://inis.iaea.org/search/searchsinglerecord.aspx?recordsFor=SingleRecord\&RN=15

$\underline{032070}$

4. https://arxiv.org/pdf/astro-ph/0303284.pdf

5. https://arxiv.org/pdf/1508.07903.pdf 
6. http://cds.cern.ch/record/608794/files/0303283.pdf

7. Available at the following site:

http://physics.gu.se/ frtbm/joomla/media/mydocs/LennartSjogren/kap6.pdf

8. Available at the following site:

https://beckassets.blob.core.windows.net/product/readingsample/442318/978019955 6885 excerpt 001.pdf

9. https://arxiv.org/pdf/astro-ph/0303283.pdf

10. Schutz B. F., "A first course in General Relativity”, Cambridge Univ. Press, 2003.

11. Available at the following site:

https://www.researchgate.net/publication/343386149 Solving the Flatness and Ho rizon Problems via Self-Organized Criticality

12. Available at the following site:

https://www.researchgate.net/publication/343614642 CMB Anomalies from SelfOrganized Criticality

13. Goldfain E., "Relativistic Cosmology and Self-Organized Criticality" (in progress). 